\title{
Lentiviral-mediated growth-associated protein-43 modification of bone marrow mesenchymal stem cells improves traumatic optic neuropathy in rats
}

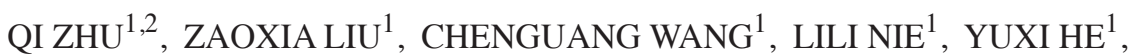 \\ YAN ZHANG ${ }^{1}$, XIN LIU ${ }^{1}$ and GUANFANG SU ${ }^{1}$ \\ ${ }^{1}$ Department of Ophthalmology, The Second Hospital of Jilin University, Changchun, Jilin 130041; \\ ${ }^{2}$ Department of Ophthalmology, Armed Police Force Hospital of Jilin, Changchun, Jilin 130058, P.R. China
}

Received September 20, 2014; Accepted June 26, 2015

DOI: $10.3892 / \mathrm{mmr} .2015 .4132$

\begin{abstract}
The aim of the present study was to examine the effect of growth-associated protein-43 (GAP-43) on bone marrow mesenchymal stem cell (BMSC) differentiation in a rat model of traumatic optic neuropathy (TON). GAP-43 and short hairpin (sh)RNA-GAP-43 were inserted into pGLV5 and pGLV3 lentiviral vectors, respectively. The stable control, GAP-43-overexpression and GAP-43-knockdown cell lines (GFP/BMSCs, GAP-43/BMSCs and shGAP-43/BMSCs, respectively) were established. The expression of GAP-43, neuron-specific enolase (NSE), nestin, neurofilament (NF), neuron-specific nuclear-binding protein (NeuN) and $\beta I I I-t u b u l i n$ were detected in the GAP-43/BMSCs and shGAP-43/BMSCs with retinal cell-conditioned differentiation medium using semi-quantitative polymerase chain reaction (PCR), western blotting and cell immunofluorescence. In addition, the BMSCs were observed under fluorescence microscopy. The Sprague-Dawley rat models of TON were established and identified by retrograde labeling of retinal ganglion cells (RGCs) with fluoroGold (FG). The lentiviral-mediated GAP-43-modified BMSCs were then transplanted into the rat model of TON. The expression of GAP-43 was detected in the retinal tissues using qPCR and western blotting. The histopathology of the retinal tissues was observed using hematoxylin and eosin (H\&E) staining. The GAP-43/BMSCs exhibited positive expression of NSE, NF, nestin and $\beta$ III-tubulin, and exhibited a neuronal phenotype. The shGAP-43/BMSCs markedly inhibited expression of NeuN, NSE, NF, nestin and $\beta$ III-tubulin induced by retinal
\end{abstract}

Correspondence to: Dr Guanfang Su, Department of Ophthalmology, The Second Hospital of Jilin University, 218 Ziqiang Road, Changchun, Jilin 130041, P.R. China

E-mail: guanfangsuu@163.com

Key words: growth-associated protein-43, genetically-modified bone mesenchymal stem cells, neural-like cells, traumatic optic neuropathy cell-conditioned differentiation medium. The FG staining revealed that the number of labeled RGCs were significantly decreased in the TON model rats, compared with normal rats $(\mathrm{P}<0.05)$. The H\&E staining revealed that the degree of pathological changes was improved in the GAP-43/BMSC group, compared with the GFP/BMSC and shGAP-43/BMSC groups. In conclusion, GAP- 43 promoted BMSC differentiation into neuron-like cells, and intravitreally injected GAP-43/BMSCs promoted the process of nerve repair in a rat model of TON.

\section{Introduction}

The number of individuals with visual impairment is estimated to be $285,000,000$ worldwide, of whom $39,000,000$ are blind (1). A variety of diseases of the eye causing blindness, including optic nerve $(\mathrm{ON})$ injury caused by trauma and glaucoma caused by optic atrophy, have no effective treatment strategies at present. Pathological changes of the ON and retinal ganglion cell (RGC) degeneration or damage are caused by various factors, which lead to the complete loss of visual function. Therefore, it is important to protect the retina and supplement retinal nerve cells in diseases of the eye, which cause blindness. Stem cell research and gene technology have been developing increasingly, which may provide novel strategies for the treatment of diseases of the eye causing blindness.

Bone marrow mesenchymal stem cells (BMSCs), a type of multipotent stem cell from the bone marrow, are capable of differentiating into a variety of lineages. Due to the ease of separation and culture, avoidance of the immune rejection and ethical issues, BMSCs have become a significant area of interest in the investigation of adult stem cells. A previous study revealed that, in the field of ophthalmology, BMSCs can be induced into corneal epithelial cells in vitro (2). Wang et al (3) demonstrated that intravitreally injected BMSCs are capable of mobilizing into cells subjected to laser-induced retinal injury and differentiating into retinal pigment epithelium, endothelial cells, pericytes and photoreceptors. Investigations using rat models subjected to ischemia/reperfusion ocular injury have demonstrated that a few BMSCs are integrated into the ganglion cell layer, following intravitreal injection, and express specific markers of neuron-specific enolase 
(NSE), neurofilament (NF) and various neurotrophic factors. Therefore, intravitreally injected BMSCs can reduce damage to RGCs (4). It is important to investigate BMSC differentiation into RG-like cells for developments of treatment of eye disease, which cause blindness, including retinitis pigmentosa, age-related macular degeneration and glaucomatous optic neuropathy (5).

Growth-associated protein-43 (GAP-43), a protein kinase $\mathrm{C}$ substrate, is a member of the calmodulin-binding protein family and concentrates in the presynaptic membrane and growth cones (6). GAP-43 is involved in neurotransmitter release, and promotes membrane expansion by vesicle fusion or inducing endocytosis of the growth cone and presynaptic terminal (7). The expression of GAP-43 is gradually increased in the process of axon regeneration (8-10). Ivanov et al (11) revealed that GAP-43 is overexpressed in adult RGCs, compared with other retinal cells using a DNA microarray method. A long-term investigation of ON injury in a zebrafish model also suggested that the phosphorylated form of GAP-43 is important in the early and late periods of nerve regeneration (10).

In the present study, the effects of lentiviral-mediated overexpression and knockdown of GAP-43 on BMSC differentiation, and the expression of phenotypic markers were investigated in vitro. In addition, a traumatic optic neuropathy (TON) rat model was established, and the effects of lentiviral-mediated GAP-43 gene-modified BMSCs on the process of nerve repair in the TON rat model were observed.

\section{Materials and methods}

Ethical statement. Approval from the Animal Ethics Committee of the Animal Laboratory Center of the Basic Medical College of Jilin University (Basic Medical College of Jilin University, Changchun, China) was obtained prior to the use of the animals in the present study.

Isolation, culture and identification of BMSCs. Fifteen healthy newborn Wistar rats (16-18 g, male), 10-days old, were provided by the Animal Laboratory Center of the Basic Medical College of Jilin University. The rats were sacrificed by cervical dislocation. BMSCs were isolated, according to previously described procedures (12). Briefly, the bone marrow was obtained from the bilateral femurs and tibias, flushed out with $5 \mathrm{ml}$ Dulbecco's modified Eagle's Medium (DMEM)/F12 (Gibco Life Technologies, Carlsbad, CA, USA) and mixed with equivalent percoll separating medium (1.073 g/l; Gibco Life Technologies). Following centrifugation at $900 \mathrm{xg}$ for $20 \mathrm{~min}$ at room temperature, the intermediate monolayer cells were collected and flushed twice with phosphate-buffered saline (PBS). The collected BMSCs were resuspended in $3 \mathrm{ml}$ DMEM/F12 containing 10\% fetal calf serum (Gibco Life Technologies) and placed into 6-well plates (Corning Incorporated, Corning, NY, USA). Following incubation for $24 \mathrm{~h}\left(37^{\circ} \mathrm{C}, 5 \% \mathrm{CO}_{2}\right.$ and saturation humidity), the non-adherent cells were removed by replacing the medium. At $>90 \%$ confluence, the cells were digested with a mixture of $0.25 \%$ Trypsin (Sigma-Aldrich, St. Louis, MO, USA) and 0.02\% EDTA (Sigma-Aldrich) and then passaged. To identify the BMSCs, cells in the third passage were collected for detection using flow cytometry. A total of $5 \times 10^{5}$ cells in $50 \mu 1 \mathrm{PBS}$ were stained with $10 \mu 1$ mouse anti-rat CD90-fluorescein isothiocyanate (FITC), CD44-FITC, CD11b-FITC and CD44-FITC monoclonal antibodies (1:50; BD Biosciences, Franklin Lakes, NJ, USA) for $30 \mathrm{~min}$ at $4^{\circ} \mathrm{C}$ in the dark, respectively. Subsequently, the cells were washed with PBS three times and detected using a FACSCalibur II flow cytometer (BD Biosciences). The data were analyzed using CellQuest software (BD Biosciences).

Preparation of lentiviral vectors. The GAP-43 gene overexpression lentivirus was constructed and termed LV5-GAP-43. The GAP-43 gene was amplified from rat cDNA. The polymerase chain reaction (PCR) program was as follows: $95^{\circ} \mathrm{C}$ for $3 \mathrm{~min}, 30$ cycles of $95^{\circ} \mathrm{C}$ for $30 \mathrm{sec}, 60^{\circ} \mathrm{C}$ for $30 \mathrm{sec}$ and $72^{\circ} \mathrm{C}$ for $90 \mathrm{sec}$, with a final elongation at $72^{\circ} \mathrm{C}$ for $10 \mathrm{~min}$ using the Geneamp PCR (Applied Biosystems, Foster City, CA, USA). Then GAP-43 gene was cloned into the pGLV5 lentiviral vector. The GAP-43 gene silencing lentiviral vector was also constructed and termed LV3-short hairpin (sh)RNA-GAP-43. A total of four target shRNAs (Gap43-rat-619, Gap43-rat-844, Gap43-rat-1215 and Gap43-rat-678) and one negative scrambled shRNA (Gap43-rat-NC), as listed in Table I, were designed using siRNA Target Finder and Design Tools software (Ambion Life Technologies, Carlsbad, CA, USA). The cloned DNA segment was inserted into the pGLV3 lentiviral vector. Sequence analysis confirmed that the inserted GAP-43 and shRNA-GAP-43 sequences were correct (Shanghai Sangon Biotech Co. Ltd., Shanghai, China). To screen for specific small interfering (si)RNAs against GAP-43, 293T cells (Sangon Biotech, Shanghai, China) were co-transfected with LV5-GAP-43 and LV3-shRNA-GAP-43-619/844/1215/678, respectively. The expression of GAP-43 was detected using western blotting and the most effective siRNAs were used for further investigation. The successfully constructed lentiviral vector and packaging plasmid (mix) were co-transfected into the 293T cells using Lipofectamine 2000 (Invitrogen Life Technologies, Carlsbad, CA, USA). Packaging and titering of the lentiviral vectors were performed, according to previously described procedures (13). After transfection for $8 \mathrm{~h}$, the culture medium was changed to complete medium. The supernatant was harvested after culturing for $48 \mathrm{~h}$ and concentrated by ultrafiltration. The virus titer was measured using the dilution gradient method and calculated as follows: Virus titer $(\mathrm{TU} / \mathrm{ml})=$ counted fluorescent cells/corresponding volume of virus stock solution. Ultimately, the titer of the lentivirus was $2 \times 10^{8} \mathrm{TU} / \mathrm{ml}$ and the lentivirus was stored at $-80^{\circ} \mathrm{C}$ for later use.

Lentivirus transduction. The third passage BMSCs were transduced with the negative control lentiviral vector, LV5-GAP-43 and LV3-shRNA-GAP-43s, with a multiplicity of infection (MOI) of 20 , and $5 \mu \mathrm{l}$ polybrene $(10 \mathrm{mg} / \mathrm{ml})$ was added, respectively. The stable control, GAP-43-overexpression and GAP-43-knockdown cell lines, termed GFP/BMSCs, GAP-43/BMSCs and shGAP-43/BMSCs, were established in culture medium containing puromycin $(10 \mu \mathrm{l} / \mathrm{ml})$.

Effects of Gap-43 on BMSC differentiation. To analyze the effects of the overexpression of GAP-43 on BMSC differentiation, the third passage BMSCs were divided into three groups: 
Table I. Short hairpin RNA sequences of GAP-43.

\begin{tabular}{|c|c|}
\hline Name & Sequence \\
\hline Gap43-rat-619 Top strand & $\begin{array}{l}\text { 5'-GATCCGCCCGACAGGATGAGGGTAAATTCA } \\
\text { AGAGATTTACCCTCATCCTGTCGGGCTTTTTTG-3' }\end{array}$ \\
\hline Gap43-rat-619 Bottom strand & $\begin{array}{l}\text { 5'-AATTCAAAAAAGCTAAAGCTACCACTGATAACT } \\
\text { CTCTTGAAGTTATCAGTGGTAGCTTTAGCG-3' }\end{array}$ \\
\hline Gap43-rat-844 Top strand & $\begin{array}{l}\text { 5'-GATCCGCCCGACAGGATGAGGGTAAATTCAAGA } \\
\text { GATTTACCCTCATCCTGTCGGGCTTTTTTG-3' }\end{array}$ \\
\hline Gap43-rat-844 Bottom strand & $\begin{array}{l}\text { 5'-AATTCAAAAAAGCCCGACAGGATGAGGGTAAAT } \\
\text { CTCTTGAATTTACCCTCATCCTGTCGGGCG-3' }\end{array}$ \\
\hline Gap43-rat-1215 Top strand & $\begin{array}{l}\text { 5'-GATCCGAGTCCACTTTCСТСТСТАTTTCAAGAG } \\
\text { AATAGAGAGGAAAGTGGACTCTTTTTTG-3' }\end{array}$ \\
\hline Gap43-rat-1215 Bottom strand & $\begin{array}{l}\text { 5'-AATTCAAAAAAGAGTCCACTTTCCTCTCTA } \\
\text { TTCTCTTGAAAGGAAAGTGGACTCG-3' }\end{array}$ \\
\hline Gap43-rat-678 Top strand & $\begin{array}{l}\text { 5'-GATCCGGAGCCTAAACAAGCCGATGTTTCA } \\
\text { AGAGAACATCGGCTTGTTTAGGCTCCTTTTTTG-3' }\end{array}$ \\
\hline Gap43-rat-678 Bottom strand & $\begin{array}{l}\text { 5'-AATTCAAAAAAGGAGCCTAAACAAGCCGATGTT } \\
\text { CTCTTGAAACATCGGCTTGTTTAGGCTCCG-3' }\end{array}$ \\
\hline Gap43-rat-NC Top strand & $\begin{array}{l}\text { 5'-GATCCGTTCTCCGAACGTGTCACGTTTCAAGAG } \\
\text { AACGTGACACGTTCGGAGAACTTTTTTG-3' }\end{array}$ \\
\hline Gap43-rat-NC Bottom strand & $\begin{array}{l}\text { 5'-AATTCAAAAAAGTTCTCCGAACGTGTCACG } \\
\text { TTCTCTTGAAACGCACGTTCGGAGAACG-3' }\end{array}$ \\
\hline
\end{tabular}

Gap43, growth-associated protein-43.

Blank, NC (transduced with negative control lentiviral vector) and GAP-43 (transduced with LV5-GAP-43). The expression levels of GAP-43, NSE, nestin, NF and $\beta$ III-tubulin were detected using semi-quantitative PCR and western blotting. The morphology of the BMSCs in each group was observed under a fluorescence microscope (Olympus Corp., Tokyo, Japan).

To analyze the effects of the silencing of GAP-43 on BMSC differentiation, the third passage BMSCs were divided into three groups: Blank, NC (transduced with negative control lentiviral vector) and shGAP-43 (transduced with LV3-shRNA-GAP-43), which were all induced by retinal cell-conditioned differentiation medium containing $10 \mathrm{ng} / \mathrm{ml}$ brain-derived neurotrophic factor (BDNF; Sigma-Aldrich) and $5 \mathrm{ng} / \mathrm{ml}$ basic fibroblast growth factor (Sigma-Aldrich). The expression levels of GAP-43, NSE, $\mathrm{NF}$, neuron-specific nuclear-binding protein (NeuN) and $\beta$ III-tubulin were detected using western blotting and cell immunofluorescence. The morphology of the BMSCs was observed under a fluorescence microscope.

Animal models of TON and BMSC transplantation. A total of 120 healthy male Sprague Dawley rats (180-200 g) were provided by the Animal Laboratory Center of the Basic Medical College of Jilin University, and acclimated to a natural day-night cycle and given free access to food and water at $21^{\circ} \mathrm{C}$ together for 1 week prior to the trial. The rats were randomly divided into five groups with 24 rats in each group. The groups were as follows: Sham group; PBS group; GFP/BMSCgroup; GAP-43/BMSC group and shGAP-43/BMSC group. The rats in each group were randomly divided into four subgroups (six rats in each subgroup): 3, 7, 14 and 28 days after surgery, respectively. The TON rat model was established using the method described by Jiang et al (14). In brief, the rats were anesthetized with $10 \%$ chloral hydrate $(3 \mathrm{ml} / \mathrm{kg}$; GE Healthcare Life Sciences, Uppsala, Sweden). An incision was made on the temporal eyelid, and the ON was exposed and isolated. The ON $2 \mathrm{~mm}$ from the eyeball was crushed using cross action forceps with a $40 \mathrm{~g}$ holding force for $30 \mathrm{sec}$. Sham surgery was performed using the same procedures but without crushing of the ON. In the GFP/BMSC, GAP-43/BMSC and shGAP-43/BMSC groups, $10^{5}$ GFP/BMSCs, GAP-43/BMSCs and shGAP-43/BMSCs were injected into the rat vitreous cavity on day $3,7,14$ and 28 post-surgery, respectively, and an equal volume of PBS was administered to the sham and PBS groups. At 5 weeks post-BMSC injection, the retinas were post-fixed in $4 \%$ paraformaldehyde (Dingguo Changsheng Biotech, Beijing, China) for $24 \mathrm{~h}$.

Retrograde labeling of RGCs with fluorogold (FG) and RGC counts. The numbers of RGCs were counted using retrograde labeled with FG. Briefly, the rats were anesthetized and their heads were immobilized. The skin overlying the skull was incised. The lambda and bregma sutures served as landmarks for drilling two holes, following which $5 \mu 15 \% \mathrm{FG}$ 
Table II. Primer sequences of GAP-43, NSE, nestin, NF, $\beta$ III-tubulin and GAPDH primers.

\begin{tabular}{ll} 
Gene & \multicolumn{1}{c}{ Primer sequence } \\
\hline GAP-43 sense & 5'-CATGAGAAGTATGACAACAGCCT-3' \\
GAP-43 antisense & 5'-AGTCCTTCCACGATACCAAAGT-3' \\
NSE sense & 5'-CAACAGCACCATCGCACCG-3' \\
NSE antisense & 5'-GGCAAAGCCGCCTTCATC-3' \\
nestin sense & 5'-CAGGCTTCTCTTGGCTTTCTGG-3' \\
nestin antisense & 5'-TGGTGAGGGTTGAGGTTTGT-3' \\
NF sense & 5'-TGGAGAATGAGCTGCGAAGC-3' \\
NF antisense & 5'-TTCGTAGCCTCAATGGTCTC-3' \\
$\beta$ IIII-tubulin sense & 5'-TGAGACCTACTGCATCGACA-3' \\
$\beta$ III-tubulin antisense & 5'-GGGATCCACTCCACGAAGTA-3' \\
GAPDH sense & 5'-CTGCAACCAAAATTCAGGCTA-3' \\
GAPDH antisense & 5'-CATCAGCAACGGGAGCAT-3'
\end{tabular}

Product size (bp)

GAP-43, growth-associated protein-43; NSE, neuron-specific enolase; NF, neurofilament.

(Sigma-Aldrich) in saline was injected into the superior colliculus once. The rats were sacrificed by cervical dislocation following injections for 7 days and the retinal tissues were harvested. The numbers of labeled RGCs were determined using an inverted fluorescence microscope (IX70; Olympus Corp.). The rate of RGC labeling was calculated as follows: RGCs from injured eye/RGCs from uninjured eye x $100 \%$.

Reverse transcription-quantitative/semi-quantitative PCR. Cells and tissues were homogenized on ice with TRIzol reagent (Invitrogen Life Technologies) and total RNA was extracted, according to the manufacturer's instructions. High-quality RNA was reverse transcribed into complementary DNA (cDNA) with a Reverse Transcription kit (cat no. DRR036A; Takara Biotechnology Co., Ltd., Dalian, China). GAP-43, NSE, nestin, NF, $\beta$ III-tubulin and glyceraldehyde-3-phosphate dehydrogenase (GAPDH) primers were used (Table II). The PCR reaction system comprised cDNA (10 ng), primers $(0.1 \mu \mathrm{M})$, deoxy-ribonucleoside triphosphate (1 mM), Taq DNA polymerase (5 U), 10X buffer $(2.5 \mu \mathrm{l})$, and double distilled water to achieve a final volume of $25 \mu 1$. PCR amplification was performed using $\mathrm{SYBR}^{\circledR}$ Premix Ex Taq $^{\text {TM }}$ (cat. no. DRR420A; Takara Biotechnology) on an ABI StepOnePlus Real-time PCR system (Applied Biosystems). The PCR protocol was as follows: $95^{\circ} \mathrm{C}$ for $2 \mathrm{~min}, 40$ cycles of $95^{\circ} \mathrm{C}$ for $10 \mathrm{sec}$ and $60^{\circ} \mathrm{C}$ for $40 \mathrm{sec}$. Relative quantification and calculations were performed using the comparative threshold $(\mathrm{Ct})$ cycle method $\left(2^{-\Delta \Delta \mathrm{Ct}}\right)$. For semi-quantitative PCR, products were analyzed using agarose gel electrophoresis and the corresponding optical density ratio was measured (optical density value of the specific gene/optical density value of GAPDH).

Western blotting. The cells were collected and lysed in radioimmunoprecipitation assay lysis buffer (Dingguo Changsheng Biotech). Supernatants were acquired by centrifugation at $12,000 \mathrm{xg}$ for $15 \mathrm{~min}$ at $4^{\circ} \mathrm{C}$. Subsequently, the protein concentration was detected using the Bicinchoninic Protein Quantitative
Assay (Shanghai Sangon Biotech). A total of $40 \mu \mathrm{g}$ protein was separated by $10 \%$ SDS-PAGE and then transferred onto polyvinylidene difluoride membranes (Amersham Pharmacia, Piscataway, NJ, USA), which were blocked in 5\% defatted milk for $1 \mathrm{~h}$ at room temperature. The membranes were incubated with rabbit anti-rat polyclonal antibodies against GAP-43, NSE, nestin, NF, $\beta$ III-tubulin and GAPDH (1:500; Wuhan Boster Biological Technology, Ltd., Wuhan, China) overnight at $4^{\circ} \mathrm{C}$, washed three times with PBS and then incubated with goat anti-mouse immunoglobulin $\mathrm{G}(\mathrm{H}+\mathrm{L})$-horseradish peroxidase (1:5,000; Wuhan Boster Biological Technology, Ltd.) for $2 \mathrm{~h}$ at room temperature. Proteins were detected by enhanced chemiluminescence (Amersham Pharmacia) and the corresponding gray value ratios (specific genes/GAPDH) were measured.

Hematoxylin and eosin $(H \& E)$ staining. The retinal tissues were harvested, fixed in $4 \%$ paraformaldehyde for $24 \mathrm{~h}$, processed and embedded into paraffin blocks. The sections of retinal tissues were dipped into gradient ethanol, stained with hematoxylin for $5 \mathrm{~min}$, differentiated in $1 \%$ hydrochloric acid alcohol for $2 \mathrm{sec}$, incubated in ammonia water for $2 \mathrm{~min}$ and then stained with eosin for $1 \mathrm{~min}$. The sections were rinsed with distilled water at each interval step. The sections were then dehydrated with gradient ethanol, cleared with xylene (Sinopharm Chemical Reagent, Beijing, China), mounted with neutral resin (Beijing Dingguo Changsheng), and observed using light microscopy (BX41; Olympus Corp.).

Cell immunofluorescence. The cells were washed twice with Hanks' balanced salt solution at $37^{\circ} \mathrm{C}$ and then fixed with $4 \%$ paraformaldehyde (precooling) for $15 \mathrm{~min}$ at $37^{\circ} \mathrm{C}$, followed by blocking in PBS containing $1 \%$ bovine serum albumin and $1 \%$ goat serum for $1 \mathrm{~h}$ at room temperature. The samples were then incubated with rabbit anti-rat nestin, NeuN and GAP-43 polyclonal antibodies (Wuhan Boster Biological Technology, Ltd.) at a dilution of 1:100 in PBS at $4^{\circ} \mathrm{C}$ overnight, washed twice with PBS and incubated with FITC- or 
A

B
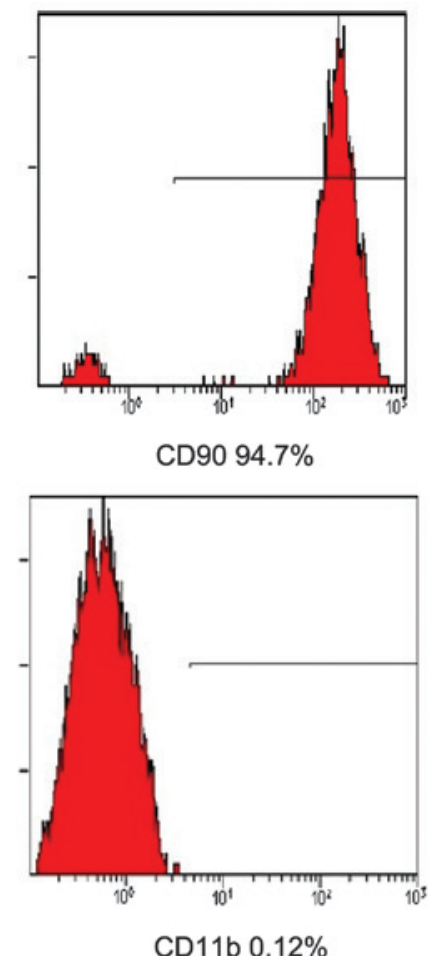
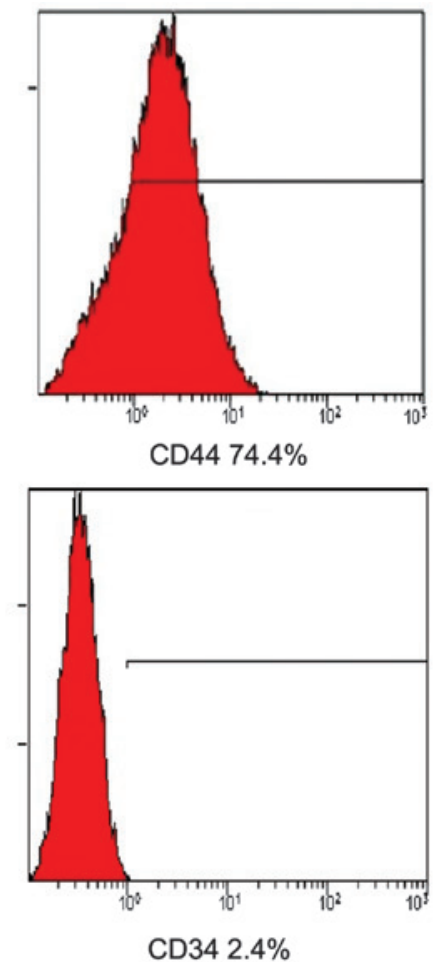

Figure 1. Morphology and identification of BMSCs using flow cytometry. (A) Third passage BMSCs uniformly assumed a vortex-like or radiating appearance. (B) Flow cytometric analysis demonstrated that third passage BMSCs were positive for CD90 and CD44, while negative for CD11b and CD34. Scale bar=50 $\mu \mathrm{m}$. BMSCs, bone marrow mesenchymal stem cells.

Cy3-labeled rabbit anti-goat IgG $(\mathrm{H}+\mathrm{L}$; Wuhan Boster Biological Technology, Ltd.) at a dilution of 1:200 in PBS at room temperature for $1 \mathrm{~h}$. Finally, images were captured using an inverted fluorescence microscope (Olympus Corp.).

Statistical analysis. Statistical analysis was performed using SPSS 12.0 statistical analysis software (SPSS, Inc., Chicago, IL, USA). The data are expressed as the mean \pm standard deviation and were analyzed using one-way analysis of variance. $\mathrm{P}<0.05$ was considered to indicate a statistically significant difference.

\section{Results}

Morphology and identification of BMSCs. The third passage BMSCs uniformly assumed a vortex-like or radiating appearance (Fig. 1A). The cells were positive for CD90 (94.7\%) and CD44 (74.4\%), and negative for CD11b (0.12\%) and CD34 (2.4\%; Fig. 1B). Therefore, the third passage BMSCs were used in the subsequent experiments.

Effects of lentiviral-mediated overexpression of GAP-43 on BMSC differentiation. Western blot analysis revealed that the protein expression of GAP-43 was significantly increased in the LV5-GAP-43 group, compared with in the $\mathrm{NC}$ and blank groups $(\mathrm{P}<0.05$; Fig. $2 \mathrm{~A})$, suggesting that the GAP-43/BMSC line was successfully established. BMSCs were observed under fluorescence microscopy 3,5 and 7 days after transduction with LV5-GAP-43. The bodies of the BMSCs were contracted with protruding processes at day 3, and the typical neuron-like cells appeared at day 5. On day 7 , the majority of the BMSCs exhibited a neuronal phenotype and the prominences formed a network structure (Fig. 2B). According to semi-quantitative PCR, the BMSCs exhibited positive expression of NSE, NF, nestin and $\beta$ III-tubulin in the LV5-GAP-43 group (Fig. 2C).

Effects of lentiviral-mediated knockdown of GAP-43 on $B M S C$ differentiation. Western blot analysis revealed that LV3-shRNA-GAP-43-619 was the most effective siRNA against GAP-43 (Fig. 3A) and was used for the subsequent investigations. Following induction by retinal cell-conditioned differentiation medium, western blot analysis demonstrated that GAP-43 was expressed in the NC and Blank groups, but not in the LV3-shRNA-GAP-43-619 group (Fig. 3B), suggesting that the shGAP-43/BMSC line was successfully established. The BMSCs exhibited a neuronal phenotype in the NC and Blank group; however, the majority of BMSCs exhibited no marked morphological changes (Fig. 3C) in the LV3-shRNA-GAP-43-619 group, suggesting that neuron-like cells were successfully induced by retinal cell-conditioned differentiation medium. The expression levels of NSE, NF, nestin and $\beta$ III-tubulin were significantly reduced in the LV3-shRNA-GAP-43-619 group, compared with the $\mathrm{NC}$ and Blank groups $(\mathrm{P}<0.05$; Fig. 3D). In addition, cell immunofluorescence analysis revealed that $\sim 80 \%$ of BMSCs expressed GAP-43, nestin and $\mathrm{NeuN}$ in the NC group under induction conditions, whereas this value was $<30 \%$ in the LV3-shRNA-GAP-43-619 group (Fig. 3E).

Effects of lentiviral-mediated GAP-43 gene-modified $B M S C$ s on a rat model of TON. FG staining revealed that 
A
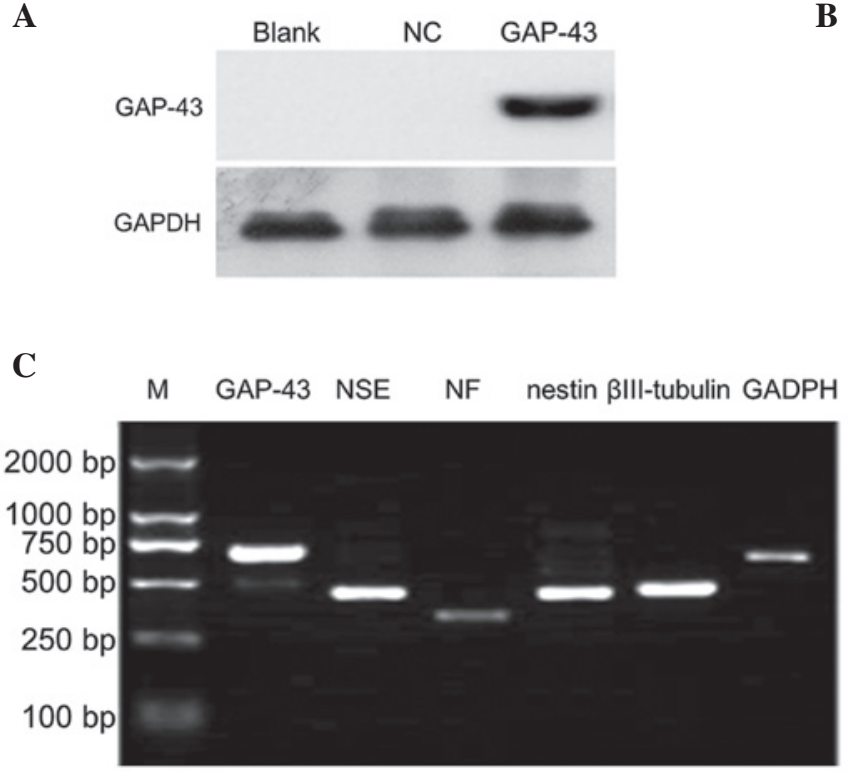

B

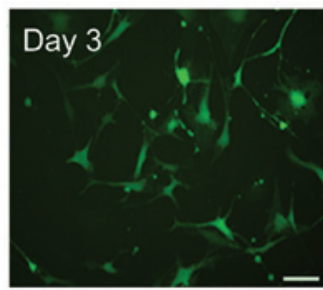

Day 5

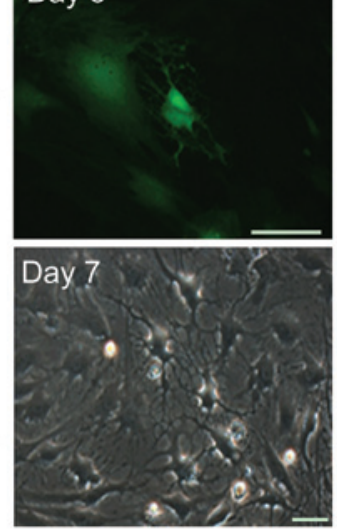

Figure 2. Overexpression of GAP-43 promotes the differentiation of BMSCs into neuron-like cells. (A) Western blot analysis demonstrated that the protein expression of the GAP-43 was significantly increased in the LV5-GAP-43 group, compared with the NC and Blank groups. (B) BMSCs were observed using microscopy and it was revealed that BMSCs exhibited a neuronal phenotype following transduction with LV5-GAP-43. (C) BMSCs expressed positive specific neural markers for NSE, NF, nestin and $\beta$ III-tubulin in the LV5-GAP-43 group, determined using semi-quantitative polymerase chain reaction detection. Scale bar=50 $\mu \mathrm{m}$. GAP-43, growth-associated protein-43; BMSCs, bone marrow mesenchymal stem cells; NSE, neuron-specific enolase; NF, neurofilament; NC, transduced with negative control scramble lentiviral vector; LV5-GAP-43, transduced with LV5-GAP-43; M, marker.

the labeled RGCs were significantly decreased in the TON model rats, compared with the normal rats $(\mathrm{P}<0.05$; Fig. $4 \mathrm{~A})$. This suggested that the TON model had been successfully established. The labeled RGCs began to reduce at day 3 and reached the lowest point at day 7 , with no further reduction with longer periods of time (Fig. 4B), suggesting that the optimum period of nerve repair was limited to 3 days. At each time-point, the mRNA level of GAP-43 in the retinal tissues was significantly increased in the GAP-43/BMSC group $(\mathrm{P}<0.01)$, compared with the GFP/BMSC group, and decreased in the shGAP-43/BMSC group, compared with the GFP/BMSC group $(\mathrm{P}<0.05$; Fig. $4 \mathrm{C})$. Similar results were observed for GAP-43 protein $(\mathrm{P}<0.05$; Fig. 4D). The retinal tissue was harvested at day 3 post-surgery (early injection) and subjected to $\mathrm{H} \& \mathrm{E}$ staining. As observed in the sections from the sham group (Fig. 4E), all layers were normally organized, the thickness of the retina was normal and no cell apoptosis was evident. In the PBS group, the outer nuclear layer of the retina became thinner, the inner nuclear layer of retina did not change and the ganglion cell layer became gradually thinner. In the GAP-43/BMSC group, all layers exhibited normal thicknesses and no significant decrease in ganglion cells was observed. The pathological changes in the GFP/BMSC group were similar to those in the GAP-43/BMSC group, with the exception that ganglion cells decreased, compared with the GAP-43/BMSC group. In the shGAP-43/BMSC group, all layers became thinner and ganglion cells exhibited marginal nuclear pyknosis, loss of nucleoli and cytoplasmic vacuolation. The degree of pathological changes was markedly improved in the GAP-43/BMSC group, compared with the GFP/BMSC and shGAP-43/BMSC groups (Fig. 4E).

\section{Discussion}

Diseases of the eye causing blindness can result in irreversible damage to retinal nerve cells (15). The present study aimed to transplant lentiviral-mediated GAP-43 gene-modified BMSCs into the rat eye of a TON model in order to compensate for the loss of retinal nerve cells. The results demonstrated that overexpression of GAP- 43 promoted the differentiation of BMSCs into neuron-like cells; however, the ability of BMSCs to differentiate into neuron-like cells was significantly weakened when the expression of GAP-43 in BMSCs was inhibited. Intravitreally injected GAP-43/BMSCs promoted the nerve repair process in the rat TON model.

In the present study, lentiviral-mediated GAP-43/shGAP-43 transfection into BMSCs was performed and the effect of GAP-43 on the differentiation of BMSCs was investigated. In previous years, investigation into the differentiation of BMSCs into nerve cells has received great attention. Experiments have demonstrated that BMSCs can be differentiated into neural cells under the action of appropriate inducers and cytokines in vitro (16). The present study demonstrated that the expression levels of specific neural markers, including NSE, nestin, $\mathrm{NF}$ and $\beta$ III-tubulin were increased in the GAP-43/BMSCs, and the morphology of the GAP-43/BMSCs was similar to that of neuronal cells, which indicated that GAP-43 induced the differentiation of BMSCs into neuron-like cells. GAP-43 is important in the developmental process of the visual nervous system (17-19) and is involved in retinal light damage repair and regeneration. Meyer et al (20) observed that GAP-43 is continuously expressed for an extended duration in mature 
A

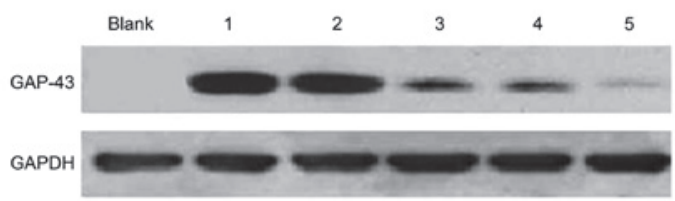

B

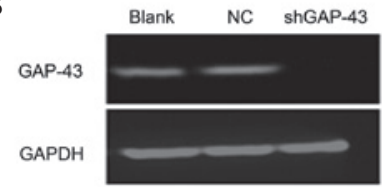

C

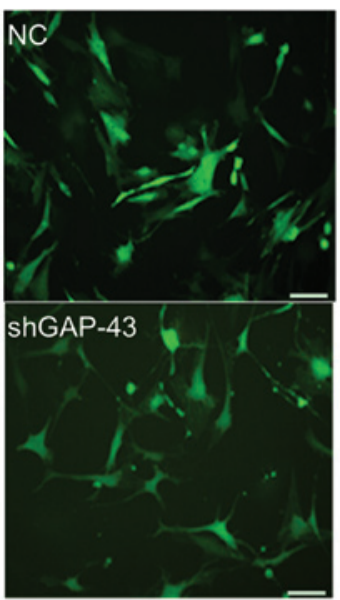

$\mathbf{E}$

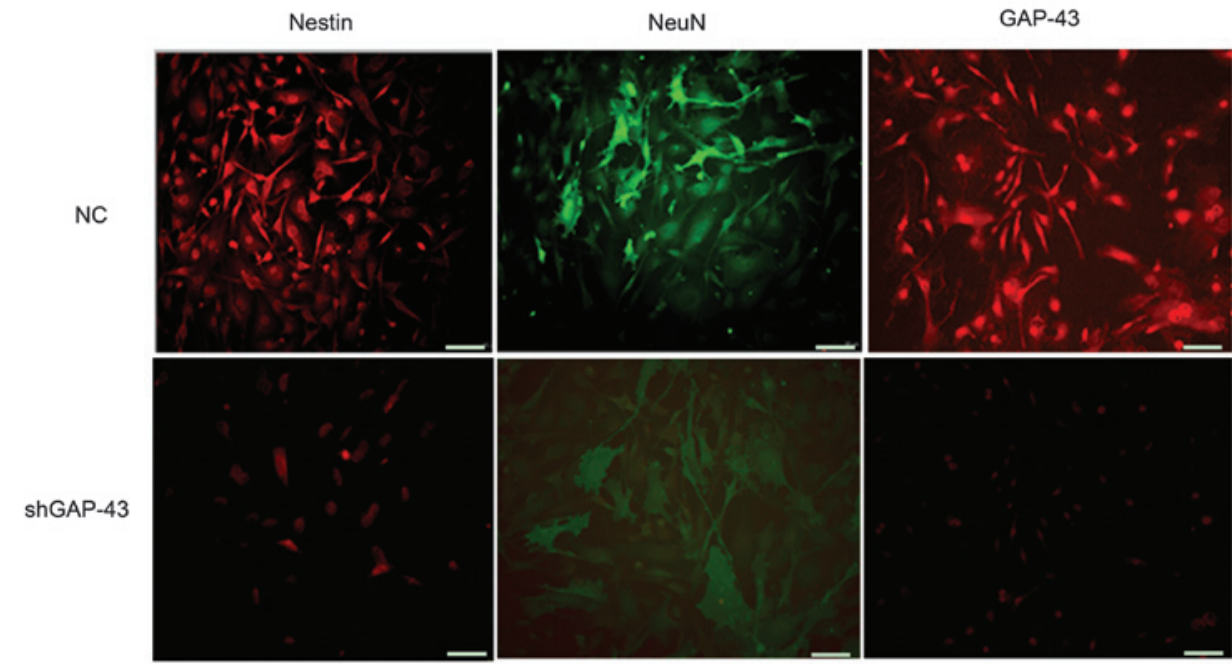

D
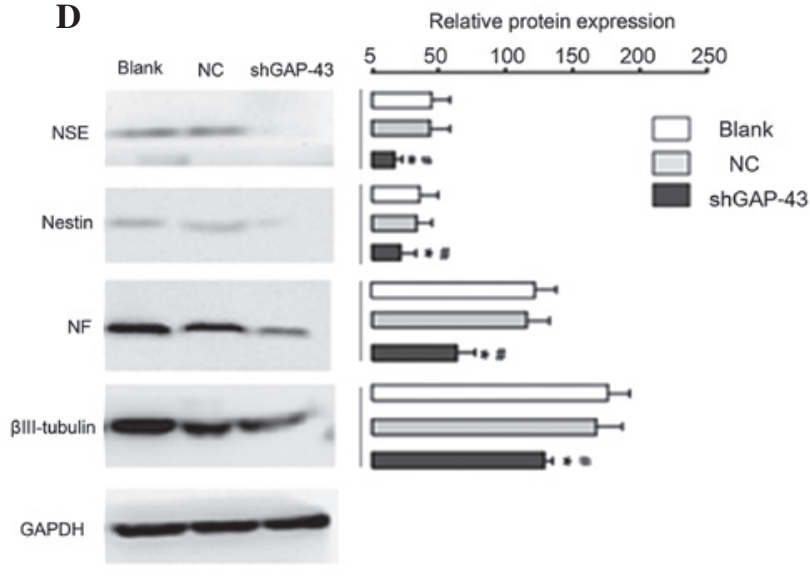

Figure 3. Ability of BMSCs to differentiate into neuron-like cells is significantly weakened when the expression of GAP-43 in BMSCs is inhibited. (A) Western blot analysis demonstrated that LV3-shRNA-GAP-43-619 was the most effective siRNA against GAP-43. 1, transduced with LV5-GAP-43; 2 , transduced with LV5-GAP-43 and LV3-shRNA-GAP-43-844; 3, transduced with LV5-GAP-43 and LV3-shRNA-GAP-43-1215; 4, transduced with LV5-GAP-43 and LV3-shRNA-GAP-43-678; 5, transduced with LV5-GAP-43 and LV3-shRNA-GAP-43-619. (B) Western blot analysis revealed that the protein expression of the GAP-43 was significantly decreased in the LV3-shRNA-GAP-43-619 group, compared with the NC and Blank groups. (C) BMSCs exhibited a neuronal phenotype in the NC and Blank groups; however, the majority of BMSCs exhibited no marked morphological changes. (D) Western blot analysis demonstrated that the expression levels of NSE, NF, nestin and $\beta$ III-tubulin were decreased in the LV3-shRNA-GAP-43-619 group compared with NC and Blank groups. (E) Cell immunofluorescence analysis revealed that the expression of GAP-43, nestin and NeuN were decreased in the LV3-shRNA-GAP-43-619 group, compared with the NC group under induction conditions. Scale bar $=50 \mu \mathrm{m} .{ }^{*} \mathrm{P}<0.05$, vs. Blank group; ${ }^{*} \mathrm{P}<0.05$, vs. NC group. GAP-43, growth-associated protein- 43 ; shRNA, short hairpin RNA; NC, transduced with negative control scramble lentiviral vector; LV3-shGAP-43, transduced with LV3-shRNA-GAP-43-619; BMSCs, bone marrow mesenchymal stem cells; siRNA, small interfering RNA; NSE, neuron-specific enolase; NF, neurofilament; NeuN, neuron-specific nuclear-binding protein.

RGCs in vitro. These results offer an explanation for the directional differentiation of GAP-43/BMSCs into neuron-like cells.

To further investigate the promoting effect of GAP-43 on ON repair, lentiviral-mediated GAP-43 gene-modified BMSCs were injected into the eye of a rat TON model at different time-points. The TON model was successfully established and defined using FG staining. It was observed that the number of RGCs decreased with the extension of survival rate. The number of RGCs began to reduce at day 3 and reached the lowest point at day 7, with no further reduction with extended duration. Thus, it was hypothesized that the loss of RGCs, caused by ON injury, might be inhibited when rats were treated the injection of neurotrophic factors or hormonal drugs within the first 3 days. However, beyond a certain range ( $>7$ or 14 days in the present study), nerve damage is irreversible. It has been reported that transplanted BMSCs survive, migrate and integrate in injured retinal tissue (21-24). A previous study has indicated that the proliferative ability of BMSCs transfected with exogenous genes remains high (25). 
A
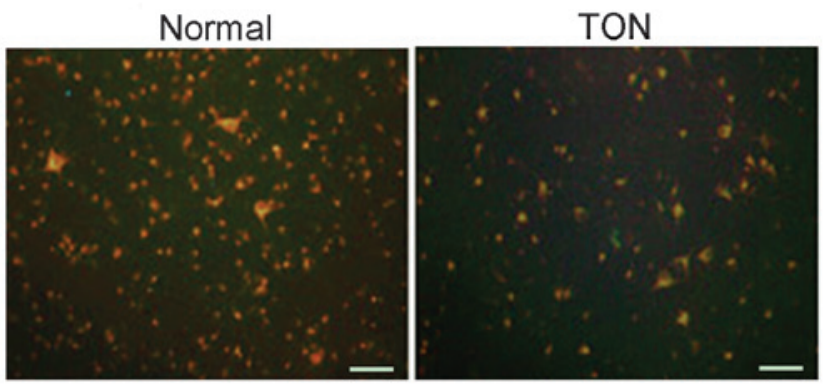

B

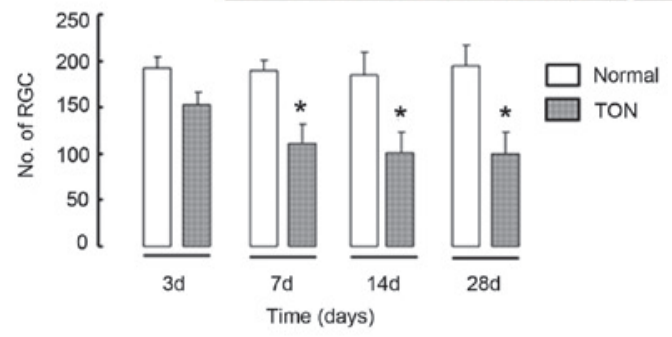

C
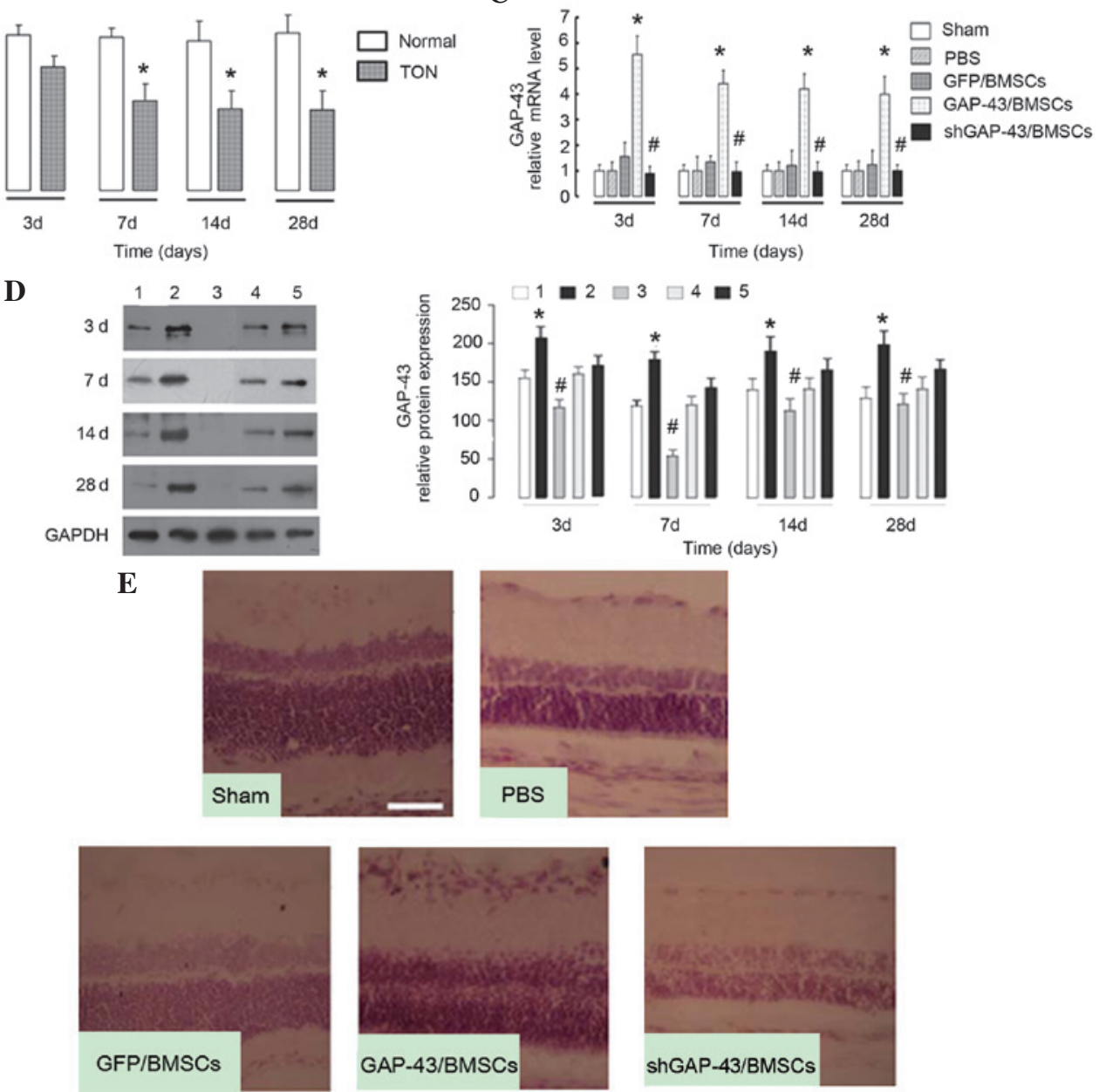

Figure 4. Intravitreally injected BMSCs overexpressing GAP-43 promote the nerve repair process in a rat model of TON. (A) Fluorogold staining revealed that the number of labeled RGCs was decreased in the TON model rats, compared with normal rats at day 7. (B) Number of labeled RGCs began to reduce at day 3 and reached the lowest point at day 7, with no further reduction as the duration extended in TON model rats, compared with the normal rats. "P<0.05, vs. normal group. (C) mRNA levels of GAP-43 in the retinal tissues was significantly increased in the GAP-43/BMSC group, compared with the GFP/BMSC control group, and decreased in the shGAP-43/BMSC group, compared with the GFP/BMSCs group at days 3, 7, 14 and 28, determined using quantitative polymerase chain reaction. (D) Protein expression of GAP-43 was significantly increased in the GAP-43/BMSC group, compared with the GFP/BMSCs group, and decreased in the shGAP-43/BMSC group, compared with the GFP/BMSCs group at days 3, 7, 14 and 28, determined using western blotting. The graph depicts the results of the densitometric quantification of the expression of GAP-43. 1, sham group; 2, GAP-43/BMSC group; 3, shGAP-43/BMSCE group; 4, PBS group; 5, GFP/BMSC group. (E) Hematoxylin and eosin staining revealed that pathological changes in the retina were markedly improved in the GAP-43/BMSC group, compared with the GFP/BMSC and shGAP-43/BMSC groups at day 3 . Scale bar $=50 \mu \mathrm{m}$. "P<0.01 GAP-43/BMSC, vs. GFP/BMSC group, ${ }^{\#} \mathrm{P}<0.05$ shGAP-43/BMSC, vs. GFP/BMSC group. GAP-43, growth-associated protein-43; shRNA, short hairpin RNA; NC, transduced with negative control scramble lentiviral vector; shGAP-43, transduced with shRNA-GAP-43-619; BMSCs, bone marrow mesenchymal stem cells; RGCs, retinal ganglion cells; TON, traumatic optic neuropathy; PBS, phosphate-buffered saline.

The present study observed that lentiviral-mediated GAP-43 was successfully expressed in the rats. Kurozumi et al (26) found that, following BDNF-modified BMSC transplantation into the brain of a rat middle cerebral artery occlusion model, the infarction area was markedly reduced and neural function exhibited a degree of recovery. In addition, Haider et al (27) also suggested that adenovirus-mediated insulin-like growth factor 1 (IGF-1) gene-modified BMSCs secrete IGF-1, and the area of myocardial infarction was significantly reduced following transplantation of gene-modified BMSCs into the rat model of myocardial infarction. These data suggest that it is feasible to transplant gene-modified BMSCs into rats. 
Notably, in the early treatment group (day 3), the degree of pathological changes was markedly improved in the GAP-43/BMSC group, compared with the GFP/BMSC and shGAP-43/BMSC groups, which indicated that intravitreally injected BMSCs overexpressing GAP-43 promoted the process of ON repair in the rat model of TON. GAP-43 is considered to be a molecular marker of neural plasticity, including nerve growth and damage repair (28-30). Ju et al (31) found that GAP-43 immunoreactivity is present in the inner plexiform layer in the normal retina; however, it appears in ganglion cells following ischemia and reperfusion, and certain ganglion cells can regenerate through the upregulation of GAP-43 in the ischemic rat retina. Vanselow et al (32) observed RGCs of embryonic and adult chickens in vitro and found that axonal forms exhibited marked differences, but expressed GAP-43. Following transplant of a segment of peripheral nerve to the vitreous body and proximal stump of the ON, regenerative RGCs expressing GAP-43 are significantly increased, which confirms there is a correlation between GAP-43 and RGC axonal regeneration $(33,34)$. Thus, the present study hypothesized that the transplantation of lentiviral-mediated GAP-43 gene-modified BMSCs into the rat eye of the TON model may compensate for the loss of RGCs and repair ON injury to a certain extent. In addition, the pathological changes in the GFP/BMSC group were similar to those in the GAP-43/BMSC group, with the exception of the decrease in ganglion cells, compared with the GAP-43/BMSCs group. Inoue et al (35) demonstrated that BMSCs injected into an area of retinal degeneration in a Royal College of Surgeons rat, successfully delayed the degeneration of the retina and preserved some function of the retina. Arnhold et al (36) suggested the introduction of adenovirally-transduced BMSCs into Wistar rats, which were able differentiate into pigment epithelial cells. These data indicated that BMSCs are involved in promoting ON repair without gene modification.

In conclusion, the present study demonstrated that GAP-43 promoted the differentiation of BMSCs into neuron-like cells, and intravitreally injected BMSCs overexpressing GAP-43 promoted the process of nerve repair in a rat model of TON. These findings suggested that lentiviral-mediated GAP-43 gene-modified BMSCs may be a valuable method for protection of the retina and from $\mathrm{ON}$ disease.

\section{Acknowledgements}

This study was supported by a grant from the General Programs of National Natural Science Foundation (grant no. 30973265$)$.

\section{References}

1. Pascolini D and Mariotti SP: Global estimates of visual impairment: 2010. Br J Ophthalmol 96: 614-618, 2012.

2. Gu S, Xing C, Han J, Tso MO and Hong J: Differentiation of rabbit bone marrow mesenchymal stem cells into corneal epithelial cells in vivo and ex vivo. Mol Vis 15: 99-107, 2009.

3. Wang HC, Brown J, Alayon H and Stuck BE: Transplantation of quantum dot-labelled bone marrow-derived stem cells into the vitreous of mice with laser-induced retinal injury: survival, integration and differentiation. Vision Res 50: 665-673, 2010.
4. Li N,Li XR and Yuan JQ: Effects of bone-marrow mesenchymal stem cells transplanted into vitreous cavity of rat injured by ischemia/reperfusion. Graefes Arch Clin Exp Ophthalmol 247: 503-514, 2009

5. Vijaya L, Asokan R, Panday M, Choudhari NS, Ramesh SV, Velumuri L, Boddupalli SD, Sunil GT and George R: Baseline risk factors for incidence of blindness in a South Indian population: The chennai eye disease incidence study. Invest Ophthalmol Vis Sci 55: 5545-5550, 2014.

6. Basi GS, Jacobson RD, Virág I, Schilling J and Skene JP: Primary structure and transcriptional regulation of GAP-43, a protein associated with nerve growth. Cell 49: 785-791, 1987.

7. Caprini M, Gomis A, Cabedo H, Planells-Cases R, Belmonte C, Viana F and Ferrer-Montiel A: GAP43 stimulates inositol trisphosphate-mediated calcium release in response to hypotonicity. EMBO J 22: 3004-3014, 2003.

8. Donovan SL, Mamounas LA, Andrews AM, Blue ME and McCasland JS: GAP-43 is critical for normal development of the serotonergic innervation in forebrain. J Neurosci 22: 3543-3552, 2002.

9. Koutcherov Y, Mai JK and Paxinos G: Hypothalamus of the human fetus. J Chem Neuroanat 26: 253-270, 2003.

10. Kaneda M, Nagashima M, Nunome T, Muramatsu T, Yamada Y, Kubo M, Muramoto K, Matsukawa T, Koriyama Y, Sugitani K, et al: Changes of phospho-growth-associated protein 43 (phospho-GAP43) in the zebrafish retina after optic nerve injury: a long-term observation. Neurosci Res 61: 281-288, 2008.

11. Ivanov D, Dvoriantchikova G, Nathanson L, Mckinnon SJ and Shestopalov VI: Microarray analysis of gene expression in adult retinal ganglion cells. FEBS Lett 580: 331-335, 2006.

12. Hiyama A, Mochida J, Iwashina T, Omi H, Watanabe T, Serigano K, Tamura F and Sakai D: Transplantation of mesenchymal stem cells in a canine disc degeneration model. J Orthop Res 26: 589-600, 2008.

13. Zeng Z, Zhang $\mathrm{C}$ and Chen J: Lentivirus-mediated RNA interference of DC-STAMP expression inhibits the fusion and resorptive activity of human osteoclasts. J Bone Miner Metab 31: 409-416, 2013

14. Jiang $\mathrm{B}$, Zhang $\mathrm{P}$, Zhou $\mathrm{D}$, Zhang $\mathrm{J}, \mathrm{Xu} \mathrm{X}$ and Tang L: Intravitreal transplantation of human umbilical cord blood stem cells protects rats from traumatic optic neuropathy. PLoS One 8: e69938, 2013.

15. Zrenner E: Will retinal implants restore vision? Science 295: 1022-1025, 2002.

16. Woodbury D, Schwarz EJ, Prockop DJ and Black IB: Adult rat and human bone marrow stromal cells differentiate into neurons. J Neurosci Res 61: 364-370, 2000.

17. Moya KL, Jhaveri S, Schneider GE and Benowitz LI: Immunohistochemical localization of GAP-43 in the developing hamster retinofugal pathway. J Comp Neurol 288: 51-58, 1989.

18. Reh TA, Tetzlaff W, Ertlmaier A and Zwiers H: Developmental study of the expression of B50/GAP-43 in rat retina. J Neurobiol 24: 949-958, 1993

19. Moya KL, Benowitz LI, Jhaveri S and Schneider GE: Changes in rapidly transported proteins in developing hamster retinofugal axons. J Neurosci 8: 4445-4454, 1988.

20. Meyer RL, Miotke JA and Benowitz LI: Injury induced expression of growth-associated protein-43 in adult mouse retinal ganglion cells in vitro. Neuroscience 63: 591-602, 1994.

21. Tomita M, Adachi Y, Yamada H, Takahashi K, Kiuchi K, Oyaizu H, Ikebukuro K, Kaneda H, Matsumura M and Ikehara S: Bone marrow-derived stem cells can differentiate into retinal cells in injured rat retina. Stem Cells 20: 279-283, 2002.

22. Kicic A, Shen WY, Wilson AS, Constable IJ, Robertson T and Rakoczy PE: Differentiation of marrow stromal cells into photoreceptors in the rat eye. J Neurosci 23: 7742-7749, 2003.

23. Yu S, Tanabe T, Dezawa M, Ishikawa $\mathrm{H}$ and Yoshimura N: Effects of bone marrow stromal cell injection in an experimental glaucoma model. Biochem Biophys Res Commun 344: 1071-1079, 2006.

24. Sengupta N, Caballero S, Mames RN, Butler JM, Scott EW and Grant MB: The role of adult bone marrow-derived stem cells in choroidal neovascularization. Invest Ophthalmol Vis Sci 44: 4908-4913, 2003.

25. Lee K, Majumdar MK, Buyaner D, Hendricks JK, Pittenger MF and Mosca JD: Human mesenchymal stem cells maintain transgene expression during expansion and differentiation. Mol Ther 3: 857-866, 2001. 
26. Kurozumi K, Nakamura K, Tamiya T, Kawano Y, Kobune M Hirai S, Uchida H, Sasaki K, Ito Y, Kato K, et al: BDNF gene-modified mesenchymal stem cells promote functional recovery and reduce infarct size in the rat middle cerebral artery occlusion model. Mol Ther 9: 189-197, 2004.

27. Haider HK, Jiang S, Idris NM and Ashraf M: IGF-1-overexpressing mesenchymal stem cells accelerate bone marrow stem cell mobilization via paracrine activation of SDF-1alpha/CXCR4 signaling to promote myocardial repair. Circ Res 103: 1300-1308, 2008.

28. Benowitz LI and Routtenberg A: GAP-43: An intrinsic determinant of neuronal development and plasticity. Trends Neurosci 20: 84-91, 1997.

29. Strittmatter SM, Vartanian T and Fishman MC: GAP-43 as a plasticity protein in neuronal form and repair. J Neurobiol 23: 507-520, 1992.

30. Dinocourt C, Gallagher SE and Thompson SM: Injury-induced axonal sprouting in the hippocampus is initiated by activation of trkB receptors. Eur J Neurosci 24: 1857-1866, 2006.

31. Ju WK, Gwon JS, Park SJ, Kim KY, Moon JI, Lee MY, Oh SJ and Chun MH: Growth-associated protein 43 is up-regulated in the ganglion cells of the ischemic rat retina. Neuroreport 13: 861-865, 2002 .
32. Vanselow J, Müller B and Thanos S: Regenerating axons from adult chick retinal ganglion cells recognize topographic cues from embryonic central targets. Vis Neurosci 6: 569-576, 1991.

33. Schaden H, Stuermer CA and Bähr M: GAP-43 immunoreactivity and axon regeneration in retinal ganglion cells of the rat J Neurobiol 25: 1570-1578, 1994.

34. Ng TF, So KF and Chung SK: Influence of peripheral nerve grafts on the expression of GAP-43 in regenerating retinal ganglion cells in adult hamsters. J Neurocytol 24: 487-496, 1995.

35. Inoue $\mathrm{Y}$, Iriyama A, Ueno S, Takahashi H, Kondo M, Tamaki Y, Araie $M$ and Yanagi Y: Subretinal transplantation of bone marrow mesenchymal stem cells delays retinal degeneration in the RCS rat model of retinal degeneration. Exp Eye Res 85: 234-241, 2007.

36. Arnhold S, Heiduschka P, Klein H, Absenger Y, Basnaoglu S, Kreppel F, Henke-Fahle S, Kochanek S, Bartz-Schmidt KU, Addicks K and Schraermeyer U: Adenovirally transduced bone marrow stromal cells differentiate into pigment epithelial cells and induce rescue effects in RCS rats. Invest Ophthalmol Vis Sci 47: 4121-4129, 2006. 